\title{
Clinical Outcomes of Various Management Strategies for Symptomatic Bradycardia
}

\author{
Victor A. Abrich, MD; Rachel J. Le, MD; Siva K. Mulpuru, MD; Paul A. Friedman, MD; \\ Gregory W. Barsness, MD; Yong-Mei Cha, MD; Ryan J. Lennon; Bradley R. Lewis; \\ and Eric H. Yang, MD
}

Objective: To determine clinical outcomes of various management strategies for reversible and irreversible causes of symptomatic bradycardia in the inpatient setting.

Design: Retrospective observational study.

Setting: Emergency room and inpatient.

Participants: Patients presenting to the emergency department with symptomatic bradycardia.

Methods: We retrospectively reviewed electronic health records of 518 patients from two Mayo Clinic campuses (Rochester and Phoenix) who presented to the emergency department with symptomatic bradycardia (heart rate $\leq 50$ beats/minute) from January I, 2010 through December 3I, 20I5. Sinus bradycardia was excluded.The following management strategies were compared: observation, non-invasive management (medications with/without transcutaneous pacing), early permanent pacemaker (PPM) implantation ( $\leq 2$ days), and delayed PPM implantation ( $\geq 3$ days). Study endpoints included length of stay and adverse events related to bradycardia (syncope, central line-associated bloodstream infections, cardiac arrest, and in-hospital mortality). Patients who received a PPM were further stratified by weekend hospital admission.

Results: Heart block occurred in 200 (38.6\%) patients, and atrial arrhythmias with slow ventricular response occurred in 239 (46.1\%) patients. Reversible causes of bradycardia included medication toxicity in $22(4.2 \%)$ patients and hyperkalemia in $44(8.5 \%)$ patients. Adverse events were similar in patients who underwent early compared to delayed PPM implantation (6.6\% vs $12.5 \%, P=.20)$, whereas adverse events were higher in patients who received temporary transvenous pacing ( $19.1 \%$ vs $3.4 \%, P<.00 \mathrm{I})$. Weekend admissions were associated with increased temporary transvenous pacing, prolonged median time to PPM implantation by I day, and prolonged median length of stay by 2 days.

Conclusions: Delayed PPM implantation was not associated with an increase in adverse events. Weekend PPM implantation should be considered to reduce temporary transvenous pacing and shorten length of stay.

Keywords: Artificial; Bradycardia; Heart Block; Pacemaker

Corresponding Author: Victor A. Abrich, MD, MercyOne Waterloo Heart Care, 2710 St. Francis Drive Ste. 320, Waterloo, IA 50702, Tel: (319) 272-5000, Fax: (319) 272-8072, Email: victor.abrich@mercyhealth.com

Financial Support: This research project received limited funding from the Division of Cardiovascular Diseases at Mayo Clinic Hospital, Phoenix, Arizona.
Received: June I, 2019

Ist revision received: August 9, 2019

2nd revision received: October 27, 2019

Accepted: December 3, 2019

doi: $10.3121 / \mathrm{cmr} .2019 .1507$ 
$\mathrm{T}$ The cardiac conduction system is prone to transient or progressive permanent dysfunction. This can lead to a decrease in heart rate with symptoms developing as a result of diminished cardiac output. Delay or block can occur at any level of the cardiac conduction system, including the sinoatrial node, the atrioventricular (AV) node, the His-Purkinje system, and the bundle branches. Symptoms include fatigue, lightheadedness, reduced functional capacity, and syncope. ${ }^{1}$ Bradycardia secondary to AV block may be due to reversible causes, including ischemia, drug toxicity (eg, beta blockers, calcium channel blockers, digoxin), and infection (eg, Lyme disease). Permanent pacemaker (PPM) implantation is often indicated in patients with dysfunction of the cardiac conduction system. Such causes may be degenerative (associated with age, hypertension, and diabetes mellitus), inflammatory (eg, sarcoidosis), infiltrative (eg, amyloidosis), metabolic (eg, hypothyroidism), and iatrogenic (eg, catheter ablation, valve surgery). ${ }^{2}$ Permanent pacemaker implantation may also be indicated in patients who require ongoing beta blocker therapy, such as those with atrial fibrillation and coronary artery disease.

Implantation rates of PPM have increased more than 50\% between 1993 and 2009, with 188,700 PPMs implanted annually in the United States alone. ${ }^{3}$ Approximately half of new device implantations are undertaken acutely, ${ }^{4}$ and implantation within 2 days of hospital admission has been associated with a low incidence of adverse events. ${ }^{5}$ Most patients presenting to the emergency department (ED) with symptomatic bradycardia and an indication for permanent pacing can be cared for in cardiac telemetry units; however, some patients may require support with temporary transvenous pacing or closer observation in locations with a higher level of care (intensive care unit [ICU], coronary care unit [CCU], or intermediate care unit). Little is known about the extent to which aggressive management of bradycardia affects clinical outcomes. Therefore, we sought to define management strategies for patients presenting with symptomatic bradycardia and to determine if these strategies had any effect on adverse events.

\section{Methods}

\section{Hospitals}

This study was conducted at two Mayo Clinic campuses: Rochester, MN, and Phoenix, AZ. The hospitals at each campus are tertiary care center teaching hospitals with 24-hour electrophysiology coverage.

\section{Study Population}

All patients who presented to a Mayo Clinic ED at the two campuses between January 1, 2010, and December 31, 2015, were evaluated for inclusion in the study. Patients were included if they had an electrocardiogram (ECG) with a ventricular rate of 50 or fewer beats per minute (bpm) and were at least 18 yearsof-age. Patients with ECGs showing sinus bradycardia were excluded to avoid including asymptomatic patients presenting to the ED for other reasons. The Mayo Clinic Institutional Review Board approved the study and waived written informed consent for those who provided research authorization.

\section{Clinical Data}

Clinical data that were abstracted included patient demographic information, comorbidities, vital signs, ECG rhythm, laboratory data, therapies, admission level of care, adverse events, length of stay, and PPM implantation date, if applicable. Laboratory data abstracted included potassium level, with hyperkalemia defined as a potassium level $\geq 6.0 \mathrm{mmol} / \mathrm{L}$. Initial ECG rhythms with documented bradycardia (ventricular rate $\leq 50 \mathrm{bpm}$ ) included second degree or higher AV block, atrial fibrillation, atrial flutter, atrial tachycardia with block, junctional rhythm, and idioventricular rhythm. Medical therapy for bradycardia included administration of atropine, dopamine, epinephrine, or isoproterenol. Reversal agents, including glucagon and digoxinspecific antibody (Digibind, GlaxoSmithKline, SpA; Parma, Italy), were also included. Patients were admitted to a hospital unit with telemetry capabilities or areas with a higher level of care (ICU, CCU, or intermediate care unit). Adverse events included syncope witnessed by staff, pauses greater than 5 seconds, cardiac arrest (ventricular tachycardia, ventricular fibrillation, asystole), all-cause mortality, and mortality attributed to bradycardia. Central line-associated bloodstream infections were also considered adverse events in patients who received a temporary transvenous pacemaker (TPM). Hospital length of stay was calculated as discharge date minus admission date.

On the basis of treatment received, four groups of patients were identified:

Observation. This group of patients did not receive a specific intervention targeted to reverse their bradycardia except they discontinued medications known to cause bradycardia or were treated for other related medical problems (eg, dialysis for hyperkalemia in patients with end-stage renal disease).

Non-invasive management. Patients in this group were treated with intravenous drugs as needed, or they received temporary transcutaneous pacing.

Early PPM implantation. Patients in this group received a PPM within 2 days of hospital admission.

Delayed PPM implantation. Patients in this group received a PPM 3 or more days after hospital admission.

In a subgroup analysis, patients who underwent PPM implantation were stratified by admission time period into weekday admissions (first ECG obtained from Monday at 7:00 AM to Friday at 5:00 PM) and weekend admissions (first ECG obtained from Friday at 5:00 PM to Monday at 7:00 AM).

\section{Statistical Analysis}

A sample size of 500 patients would be sufficient to provide $80 \%$ statistical power to detect a standardized effect size of $15 \%$ between four groups at a $5 \%$ significance level, assuming an equal number of patients in each group. Categorical variables were expressed as percentages, whereas continuous variables were expressed as mean \pm standard deviation (SD) or median (interquartile range) for skewed data. Comparison of categorical variables was performed using the $\chi^{2}$ test. Comparison of continuous variables from two groups was 


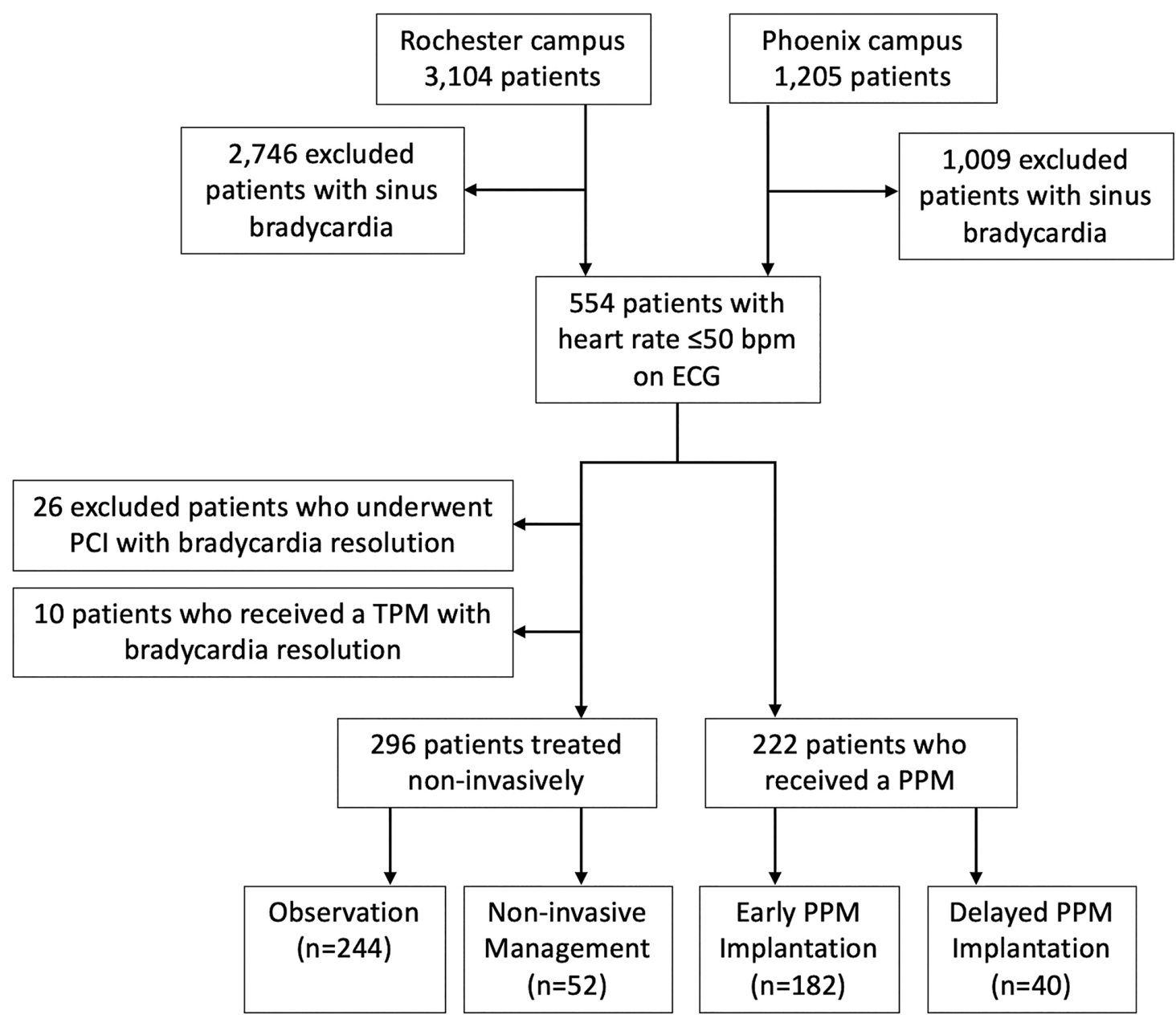

Figure 1. Patient screening for study inclusion. [ECG, electrocardiogram; bpm, beats per minute; $\mathrm{PCl}$, percutaneous coronary intervention; PPM, permanent pacemaker; TPM; temporary transvenous pacemaker].

performed using a 2-tailed t test, whereas comparison of four groups was performed using a 1-way analysis of variance (ANOVA) test. For nonparametric variables, the Wilcoxon rank sum test was used for comparison of two groups, whereas the Kruskal-Wallis test was used for comparison of four groups. Due to testing of multiple variables in these four groups of patients, correction for false discovery rate was performed using the Benjamini-Hochberg procedure using an assumed false discovery rate of 0.05 . The reported $P$ values in Tables 1 and 2 have been adjusted based on this correction. A $P$ value of $<.05$ was considered statistically significant. Statistical analyses were performed using JMP Statistical Discovery software, version 10 (SAS Institute Inc, Cary, NC).

\section{Results}

\section{Cohort Derivation}

A total of 4,310 patients between the two sites were screened for inclusion in the study (Figure 1). After excluding all patients with sinus bradycardia, 554 patients remained. Of these, 26 patients who had an acute coronary syndrome and underwent percutaneous coronary intervention with subsequent resolution of their bradycardia were excluded. Additionally, there were also 10 patients who initially required hemodynamic support with a TPM; however, after a period of monitoring in a higher level of care, the bradycardia resolved and the TPM was removed. Despite an invasive approach, these patients did not ultimately undergo PPM implantation, since they were deemed to have reversible causes of bradycardia. Since they represented a minority of patients, these 10 patients were excluded to simplify the analysis. This left a cohort of 518 patients, which was divided into the four aforementioned groups on the basis of treatment received.

\section{Demographic Characteristics, Clinical Presentation, and Comorbidities}

The mean age of the total cohort was $78.7 \pm 12.2$ years, with most patients being white $(499,96.3 \%)$ and male (307, $59.2 \%)$. The observation group was the largest of the four groups, comprising $47.1 \%$ of the total cohort (244 patients). A PPM was implanted in $222(42.9 \%)$ patients, of which 181 $(81.5 \%)$ were in the early PPM implantation group. The observation group was associated with a significantly higher 
Table 1. Demographic and Clinical Characteristics

\begin{tabular}{|c|c|c|c|c|c|}
\hline Characteristic & $\begin{array}{l}\text { Observation } \\
(n=244)\end{array}$ & $\begin{array}{l}\text { Non-invasive } \\
\text { Management } \\
(\mathrm{n}=52)\end{array}$ & $\begin{array}{l}\text { Early PPM } \\
\text { Implantation } \\
(n=182)\end{array}$ & $\begin{array}{l}\text { Delayed PPM } \\
\text { Implantation } \\
(n=40)\end{array}$ & $\begin{array}{l}\text { Adjusted } \\
P \text { Value }\end{array}$ \\
\hline Age, mean $\pm S D$, years & $79.5 \pm 11.6$ & $75.3 \pm 15.9$ & $78.7 \pm 11.7$ & $80.1 \pm 11.7$ & .18 \\
\hline Men, No. (\%) & $152(62.3)$ & $24(46.2)$ & $103(56.6)$ & $28(70.0)$ & .08 \\
\hline White, No. (\%) & $233(95.5)$ & $47(90.4)$ & $179(98.4)$ & $40(100.0)$ & .03 \\
\hline Heart rate nadir in ED, mean $\pm S D$, bpm & $43.2 \pm 7.2$ & $37.8 \pm 8.5$ & $36.3 \pm 7.1$ & $38.0 \pm 9.1$ & $<.001$ \\
\hline $\begin{array}{l}\text { Systolic blood pressure nadir in ED, mean } \pm \\
\text { SD, } \mathrm{mm} \mathrm{Hg}\end{array}$ & $118.3 \pm 26.6$ & $88.4 \pm 27.5$ & $127.4 \pm 26.5$ & $121.0 \pm 36.0$ & $<.001$ \\
\hline Medication toxicity, No. (\%) & $10(4.1)$ & $12(23.1)$ & $0(0.0)$ & $0(0.0)$ & $<.001$ \\
\hline Hyperkalemia, No. (\%) & $27(11.1)$ & $14(26.9)$ & $2(1.1)$ & $1(2.5)$ & $<.001$ \\
\hline Admission to a higher level of care, ${ }^{a}$ No. (\%) & $48(19.6)$ & $43(82.6)$ & $90(49.4)$ & $22(55.0)$ & $<.001$ \\
\hline Temporary transcutaneous pacing, No. (\%) & $\mathrm{N} / \mathrm{A}$ & $11(21.2)$ & $7(3.8)$ & $5(12.5)$ & $<.001$ \\
\hline Temporary transvenous pacing, No. (\%) & $\mathrm{N} / \mathrm{A}$ & $\mathrm{N} / \mathrm{A}$ & $35(19.2)$ & $12(30.0)$ & .16 \\
\hline Length of stay, median (IQR), days & $2.0(1.0-4.0)$ & $3.5(2.0-6.0)$ & $2.0(1.8-3.0)$ & $5.5(4.0-7.8)$ & $<.001$ \\
\hline AV block, No. (\%) & $37(15.2)$ & $6(11.5)$ & $137(75.3)$ & $20(50.0)$ & $<.001$ \\
\hline $\begin{array}{l}\text { Atrial arrhythmias with slow ventricular } \\
\text { response, }{ }^{b} \text { No. (\%) }\end{array}$ & $165(67.6)$ & $25(48.1)$ & $32(17.6)$ & $17(42.5)$ & $<.001$ \\
\hline Hypertension, No. (\%) & $171(70.1)$ & $36(69.2)$ & $145(79.7)$ & $34(85.0)$ & .06 \\
\hline Coronary artery disease, No. (\%) & $94(38.5)$ & $17(32.7)$ & $55(30.2)$ & $20(50.0)$ & .04 \\
\hline Diabetes mellitus, No. (\%) & $98(40.2)$ & $15(28.8)$ & $45(24.7)$ & $19(47.5)$ & .004 \\
\hline $\begin{array}{l}\text { History of stroke or transient ischemic attack, } \\
\text { No. }(\%)\end{array}$ & $63(25.8)$ & $15(28.8)$ & $28(15.4)$ & $12(29.3)$ & 0.04 \\
\hline Atrial fibrillation or atrial flutter, No. (\%) & $170(69.7)$ & $33(63.5)$ & 69 (37.9) & $26(65.0)$ & $<.001$ \\
\hline Chronic kidney disease, No. (\%) & $106(43.4)$ & $23(44.2)$ & $47(25.8)$ & $22(55.0)$ & $<.001$ \\
\hline Peripheral vascular disease, No. (\%) & $10(4.1)$ & $5(9.6)$ & $7(3.8)$ & $4(10.0)$ & .18 \\
\hline Congestive heart failure, No. (\%) & $117(48.0)$ & $26(50.0)$ & $62(34.1)$ & $21(52.5)$ & .02 \\
\hline Presence of $\geq 5$ comorbidities, No. (\%) & $80(32.8)$ & $15(28.8)$ & $29(15.9)$ & $15(37.5)$ & $<.001$ \\
\hline \multicolumn{6}{|c|}{$\begin{array}{l}\text { Abbreviations: AV, atrioventricular; bpm, beats per minute; ED, emergency department; ECG, electrocardiogram; IQR, interquartile } \\
\text { range; N/A, not applicable; PPM, permanent pacemaker; SD, standard deviation. } \\
\text { a Indicates admission to an intensive care unit, a coronary care unit, or an intermediate care unit. } \\
\text { b Includes atrial fibrillation, atrial flutter, and atrial tachycardia with block. }\end{array}$} \\
\hline
\end{tabular}

mean heart rate than the other groups $(P<.001)$, and the non-invasive management group had the lowest mean systolic blood pressure $(P<.001)$ (Table 1$)$.

Of the total patients, 66 had reversible causes of bradycardia: 44 (8.5\%) patients had hyperkalemia; and $22(4.2 \%)$ patients presented with medication toxicity, $8(1.5 \%)$ of whom were given a reversal agent (glucagon or digoxin-specific antibody). Admission to a higher level of care occurred in $82.6 \%$ of patients in the non-invasive management group, $19.6 \%$ of patients in the observation group, and $50.5 \%$ of patients who received a PPM $(P<.001)$. Of these admissions, $16.7 \%$ went to an intermediate care unit. The shortest median length of stay was seen in the observation and early PPM implantation groups ( 2 days, $P<.001$ ). The delayed PPM implantation group had the highest proportion of patients with more than five comorbidities at $37.5 \%$, whereas the early PPM implantation group had the lowest proportion of such patients at $15.9 \%(P<.001)$.

\section{ECG Characteristics}

Rhythms related to heart block (second degree Mobitz type I, second degree Mobitz type II, 2:1, third degree, and high grade) occurred in $200(38.5 \%)$ patients, whereas atrial arrhythmias with slow ventricular response (atrial fibrillation, atrial flutter, and atrial tachycardia with block) occurred in 239 (46.1\%) patients. Rhythms related to heart block comprised the largest proportion of the early PPM implantation group $(75.3 \%$, $P<.001)$, whereas atrial arrhythmias with slow ventricular response comprised the largest proportion of the observation group $(67.6 \%, P<.001)$ (Table 1$)$. 
Table 2. Adverse events

\begin{tabular}{|c|c|c|c|c|c|}
\hline Adverse Event & $\begin{array}{l}\text { Observation } \\
(n=244)\end{array}$ & $\begin{array}{l}\text { Non-invasive } \\
\text { Management } \\
(\mathrm{n}=52)\end{array}$ & $\begin{array}{l}\text { Early PPM } \\
\text { Implantation } \\
(\mathrm{n}=182)\end{array}$ & $\begin{array}{l}\text { Delayed PPM } \\
\text { Implantation } \\
(n=40)\end{array}$ & $\begin{array}{l}\text { Adjusted } \\
P \text { value }\end{array}$ \\
\hline Syncope, No. (\%) & $1(0.4)$ & $3(5.8)$ & $8(4.4)$ & $2(5.0)$ & .03 \\
\hline $\begin{array}{l}\text { Central line-associated bloodstream } \\
\text { infection, No. (\%) }\end{array}$ & $0(0.0)$ & $0(0.0)$ & $0(0.0)$ & $1(2.5)$ & N/A \\
\hline Cardiac arrest, No. (\%) & $2(0.8)$ & $3(5.8)$ & $4(2.2)$ & $2(5.0)$ & .11 \\
\hline $\begin{array}{l}\text { Mortality attributable to bradycardia, } \\
\text { No. }(\%)\end{array}$ & $0(0.0)$ & $3(5.8)$ & $0(0.0)$ & $0(0.0)$ & N/A \\
\hline $\begin{array}{l}\text { Total adverse events related to } \\
\text { bradycardia, No. (\%) }\end{array}$ & $3(1.2)$ & $9(17.3)$ & $12(6.6)$ & $5(12.5)$ & $<.001$ \\
\hline
\end{tabular}

\section{Outcomes}

There were 29 adverse events related to bradycardia. Overall, adverse events occurred in a larger proportion of patients who were admitted to a higher level of care than in patients admitted to the telemetry unit $(14.5 \%$ vs $6.9 \%, P<.001)$. The non-invasive management group had the highest proportion of patients with adverse events related to bradycardia $(17.3 \%$, $P<.001$ ) (Table 2). In patients who received a PPM, the incidence of adverse events was not significantly different between the early and delayed PPM implantation groups $(6.6 \%$ vs $12.5 \%, P=.20)$. Additional support by a TPM was associated with a higher proportion of patients with adverse events $(19.1 \%$ vs $3.4 \%, P<.001)$. There were 16 total deaths, 15 of which were in the observation and non-invasive management groups. There were three deaths attributed to bradycardia in the non-invasive management group after these patients declined recommended therapy. Five deaths were attributed to respiratory failure, four deaths were attributed to infection, two deaths were attributed to stroke, and two deaths were attributed to vasodilatory shock.

Of the 222 patients who received a PPM, 57 (25.7\%) patients were admitted to the hospital during weekend hours (Table 3).
Delayed PPM implantation and increased TPM usage were observed in a significantly larger proportion of these patients than in those admitted during weekday hours. Patients admitted on weekends had a longer median time to PPM implantation by 1 day $(P<.001)$ and longer median length of stay by 2 days $(P<.001)$. Adverse events were similar in patients who were admitted during weekend hours compared with weekday hours $(12.3 \%$ vs $4.8 \%, P=.05)$. This was also the case when considering only those patients who received a TPM beforehand (27.8\% vs. $13.8 \%, P=.24)$.

\section{Discussion}

Patients may be hospitalized for bradycardia for various causes, and severity ranges from reversible causes that can be managed non-invasively for patients in a telemetry unit to irreversible causes that may require temporary transvenous pacing in an ICU before PPM implantation. The non-invasive management group had the highest proportion of patients admitted to a higher level of care. This is likely because this group had the highest proportion of patients treated for medication toxicity as well as hyperkalemia. This group also included patients who died from causes unrelated to bradycardia, which likely also influenced their admission to a higher level of care. Not surprisingly, more

Table 3. Comparison of weekend and weekday admission in patients who received a permanent pacemaker.

\begin{tabular}{|c|c|c|c|}
\hline \multirow[b]{2}{*}{ Factor } & \multicolumn{2}{|c|}{ Admission } & \multirow[b]{2}{*}{$P$ value } \\
\hline & Weekend $(n=57)$ & Weekday $(n=165)$ & \\
\hline Delayed implantation, No. (\%) & $17(29.8)$ & $23(13.9)$ & .007 \\
\hline Received temporary transvenous pacing, No. (\%) & $18(31.6)$ & $29(17.6)$ & .03 \\
\hline Patients with adverse events, No. (\%) & $7(12.3)$ & $8(4.8)$ & .05 \\
\hline AV block, No. (\%) & $40(70.2)$ & $117(70.9)$ & .92 \\
\hline Admission to a higher level of care, No. (\%) & $35(61.4)$ & $77(46.7)$ & .05 \\
\hline Time to PPM implantation, median (IQR), days & $2(1-3)$ & $1(0-1)$ & $<.001$ \\
\hline Length of stay median (IQR), days & $4(3-5)$ & $2(1.5-4)$ & $<.001$ \\
\hline
\end{tabular}

Abbreviations: AV, atrioventricular; bpm, beats per minute; ECG, electrocardiogram; ED, emergency department; IQR, interquartile range; PPM, permanent pacemaker; SD, standard deviation. 
patients admitted to a higher level of care had adverse events compared to patients admitted to the telemetry unit. The proportion of patients with more than five comorbidities was much higher in patients who underwent delayed PPM implantation compared to patients who underwent early PPM implantation. It is possible that medical stabilization of comorbid conditions may have contributed to the delay in implanting a PPM in these sicker patients. Weekend admissions were associated with higher TPM usage, as well as prolonged the median time to PPM implantation and median length of stay without an increase in adverse events. Mortality was highest in the observation and non-invasive management groups and was mostly attributable to infection or respiratory failure.

The majority of patients with reversible causes of bradycardia attributed to medication overdose or hyperkalemia had their care managed either by observation or non-invasive management. Patients with medication toxicity most commonly presented either with atrial arrhythmias with slow ventricular response or junctional bradycardia, likely due to increased AV nodal blockade from either supratherapeutic medication effect and/or progressive AV conduction disturbance, with or without underlying sinus node dysfunction. In contrast, rhythms known to be associated with hyperkalemia include junctional bradycardia, second-degree heart block, and third-degree heart block, which are reversible with correction of the potassium level. ${ }^{6}$ In our study, these rhythms were all represented among patients with hyperkalemia.

Patients who had irreversible causes of bradycardia were referred for a PPM, most undergoing PPM implantation within 2 days. There were multiple reasons for deferring PPM implantation in the remaining patients, the most common being medical stabilization of patients too sick to go for an emergent procedure, drug holiday to check for reversible causes of bradycardia, infection, congestive heart failure, hyperkalemia, and elevated international normalized ratio. Adverse events were similar between patients who underwent early vs delayed PPM implantation, with no deaths attributed to a bradycardia cause. This is in contrast to other studies that have shown a higher incidence of adverse events with prolonged time to PPM implantation. A Canadian study of 214 patients who underwent urgent/emergent PPM implantation reported a mean time to PPM implantation of 1.9 days at one hospital with a dedicated procedure room and 4.5 days at a second hospital where implantation occurred in an operating room, a difference of 2.6 days. ${ }^{5}$ Adverse events during the waiting period were higher at the second hospital, particularly infection and TPM-related complications. In a Danish study that included 259 patients requiring urgent PPM implantation, the mean time to PPM implantation from referral was 5.1 days, mainly attributed to lack of implantation capacity relative to demand. ${ }^{4}$ In this study, third-degree heart block was predictive of severe adverse events, such as cardiac arrest, syncope, and ventricular tachycardia. Infections were more prevalent in patients with hospital stays extending beyond 6 days.
Before PPM implantation, some patients in our study required temporary transvenous pacing during their hospital admission. A higher percentage of patients who received a TPM had adverse events than patients who did not have a TPM. This finding could be a reflection of a cohort of patients requiring more aggressive pacing support; however, it could also be a result of complications associated with TPM use. These include loss of capture leading to pauses and syncope, as well as infection, which occurred in only one patient.

Our study also evaluated the impact of weekend hospital admission for patients who received a PPM. The percentage of patients that suffered adverse events was similar between patients admitted during weekend and weekday hours, although they had a longer median time to PPM implantation (by 1 day) and median length of stay (by 2 days). This is most likely a result of PPM implantation being deferred until regular business hours, when the electrophysiology laboratories were fully staffed and operational. A longer time to PPM implantation may also be a contributing factor for TPM support being used in more patients admitted during the weekend. PPM implantation can be performed during weekend hours at the discretion of the implanting electrophysiologist; however, this may only be feasible if 24/7 electrophysiology service coverage is available. This could potentially lead to shorter length of stay and result in substantial hospital cost savings. ${ }^{7}$

Our study had a few limitations. Because this was a retrospective study assessing different management strategies of bradycardia, the patient population was heterogeneous. We were not able to control for the various reasons that patients had delayed PPM implantation. The cause of bradycardia was unable to be accurately determined in a number of patients. For example, some patients treated for medication toxicity may have had another primary cause of bradycardia. Along the same line, the ideal treatment strategy for each individual patient may not have been evident to the treating physician at the time of presentation. This could have potentially prolonged the hospital length of stay in some patients. Hospital size and practice differences among physicians and between campuses may have also influenced the treatment provided. Additionally, scheduling and daytime availability in the electrophysiology laboratory could have influenced the timing of PPM implantations. Differentiating early PPM implantations due to urgent need from those due to increased laboratory availability was not feasible.

\section{Conclusion}

Patients can present with a variety of bradyarrhythmias. It is important to identify and treat those that are reversible. Many patients have irreversible dysfunction and require PPM implantation, some of whom may require temporary transvenous pacing. PPM implantation within 2 days can be performed in the majority of patients when medically feasible; however, delaying PPM implantation may be reasonable and necessary in several clinical situations, which in our study was not associated with an increase in adverse events. Although 24/7 electrophysiology 
services coverage may not be available in most hospitals, weekend PPM implantation should be considered in certain cases to reduce the need for temporary transvenous pacing, shorten length of stay, and reduce the cost of hospitalization.

\section{Acknowledgements}

We would like to thank Kristin E. Karlson and Edith Andrist for supplying the electrocardiographic data used in this study.

\section{References}

1. Samii SM. Indications for pacemakers, implantable cardioverterdefibrillator and cardiac resynchronization devices. Med Clin North Am. 2015;99(4):795-804.

2. Kusumoto FM, Schoenfeld MH, Barrett C, et al. 2018 ACC/ AHA/HRS Guideline on the Evaluation and Management of Patients With Bradycardia and Cardiac Conduction Delay: A Report of the American College of Cardiology/American Heart Association Task Force on Clinical Practice Guidelines and the Heart Rhythm Society. J Am Coll Cardiol. 2019;74(7):e51-e156.

3. Greenspon AJ, Patel JD, Lau E, et al. Trends in permanent pacemaker implantation in the United States from 1993 to 2009: increasing complexity of patients and procedures. J Am Coll Cardiol. 2012;60(16):1540-1545.

4. Risgaard B, Elming H, Jensen GV, Johansen JB, Toft JC. Waiting for a pacemaker: is it dangerous? Europace. 2012;14(7):975-980.

5. Simpson CS, Fisher MA, Curtis MJ, et al. Correlation of waiting time with adverse events in patients admitted for nonelective permanent pacemaker implantation. Can J Cardiol. 1998;14(6):817-821.

6. Parham WA, Mehdirad AA, Biermann KM, Fredman CS. Hyperkalemia revisited. Tex Heart Inst J. 2006;33(1):40-47.

7. Osman F, Krishnamoorthy S, Nadir A, Mullin P, Morley-Davies A, Creamer J. Safety and cost-effectiveness of same day permanent pacemaker implantation. Am J Cardiol. 2010;106(3):383-385.

\section{Author Affiliations}

Victor A. Abrich, MD*, Rachel J. Le, MD†, Siva K. Mulpuru, MD‡, Paul A. Friedman, MDt, Gregory W. Barsness, MDt, Yong-Mei Cha, MDF, Ryan J. Lennonๆ, Bradley R. Lewis ๆ, Eric H. Yang, MD*

*Division of Cardiovascular Diseases, Mayo Clinic Hospital, Phoenix, Arizona, USA; Current affiliation: MercyOne Waterloo Heart Care, Waterloo, Iowa, USA

†Providence Spokane Cardiology, Spokane, Washington, USA

$\$$ Division of Cardiovascular Diseases, Mayo Clinic, Rochester, Minnesota, USA

qDivision of Biomedical Statistics and Informatics, Mayo Clinic, Rochester, Minnesota, USA 